\title{
LONG-TERM PREDICTION OF BEACH PROFILE AND SEDIMENT GRAIN SIZE CHARACTERISTIC AT LOW ENERGY BEACH
}

\author{
Gozo Tsujimoto ${ }^{1}$, Masahiro Tamai ${ }^{2}$,Fumihiko Yamada ${ }^{3}$
}

\begin{abstract}
Artificial sandy beach profiles and vertical distributions of sediment grain size were measured monthly along two crossshore lines at Azure Maiko Beach for 2 years. All measured profiles and vertical distributions are approximated using quadratic profiles. To interpret temporal variations of these profiles, six parameters are introduced. Principle component analysis was applied to evolution of the six parameters to detect major variation modes. Relationships between the detected modes and external forces, wave height and tidal level are expressed using multiple linear regression analysis. The first mode was found to be caused by tidal oscillations and the second by energetic wave motions. Long-term prediction of the modes is examined using these regression results.
\end{abstract}

Keywords: principal component analysis; quadratic polynomial equations

\section{Introduction}

It is generally known that the greatest beach profile changes occur along the ocean side. Therefore many studies have focused on open coast beaches in high-energy environments. Wave energy and sediment transport rates on sandy beaches in very sheltered locations are much smaller than those on ocean side beaches. Such a beach is termed a "low-energy beach", and characterized by calm conditions and minimal non-storm wave heights (H1/3 less than $0.25 \mathrm{~m}$ ). Larger wave heights (H1/3 less than $0.5 \mathrm{~m}$ ) occur during storms that drive onshore winds (Jackson et al., 2002). Some studies of low-energy beaches point out differences in the characteristics and nature of beach profile response to high energy beaches. Nonetheless, low-energy beaches have generally been subsumed within existing policies that manage high-energy beaches. Therefore, a prediction method for beach profile changes and sediment grain size distributions of low-energy beaches is necessary.

In this study, sandy beach profiles and vertical distributions of sediment grain size were measured monthly along two cross-shore lines at Azure Maiko Beach for two years. A statistical method was used to analyze beach profiles and grain size distributions. The main goals were: (1) to demonstrate that the relationship between beach profile and sediment distribution is divided into two modes; (2) to simulate temporal variations of both modes.

\section{Field measurements}

Azure Maiko Beach is a micro-tidal, barrier-enclosed artificial sandy beach of about $710 \mathrm{~m}$ length, adjacent to the city of Kobe (Fig.1). Hence, wave conditions are steady and calm. Cross-shore beach profiles and sediment grain size on the foreshore have been measured monthly since February 2009. Grain size is estimated from digital images using the autocorrelation method of Rubin (2004).

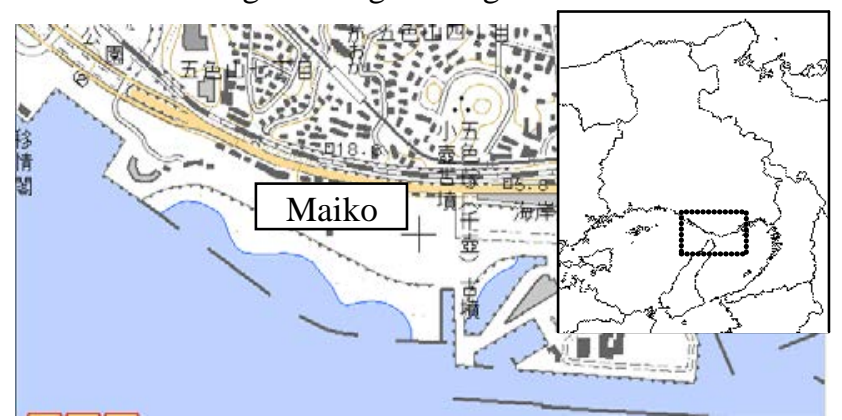

Fig. 1 Field site location

\footnotetext{
${ }^{1}$ Civil Engineering, Kobe City College of Technology, 8-3 Gakuenhigashimachi, NIshiku, Kobe 651-2194,Japan

${ }^{2}$ Civil Engineering, Osaka University, 8-3 Gakuenhigashimachi, NIshiku, Kobe, 651-2194 Japan

${ }^{3}$ Civil Engineering, Kumamoto University, 8-3 Gakuenhigashimachi, NIshiku, Kobe 651-2194,Japan
} 
Figure 2 shows measured cross-shore beach profiles along the survey line, from February 2009 to January 2011. The survey line length is not constant, and depends on tidal level changes. The range of temporal fluctuations of the measured beach profiles is approximately $0.5 \mathrm{~m}$.

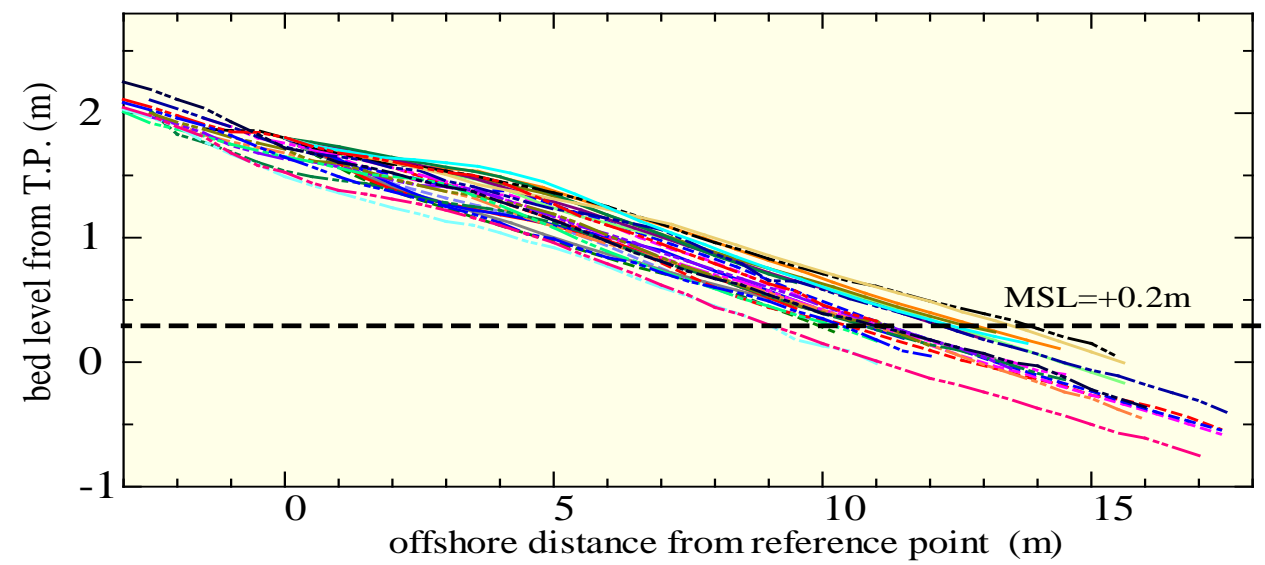

Fig. 2 Cross-shore beach profiles

Pictures of the bed surface were taken with a digital camera at several-millimeters intervals, and average particle size over a range of $\sim 10 \mathrm{~cm}$ from the outer layer was calculated. The grain size distributions are shown in Fig.3. The right edge of the survey line is the shoreline. Variations of grain size are remarkable. There are two grain size peaks along the survey line; the first is located at the final breaking point and the second at the maximum run-up point.

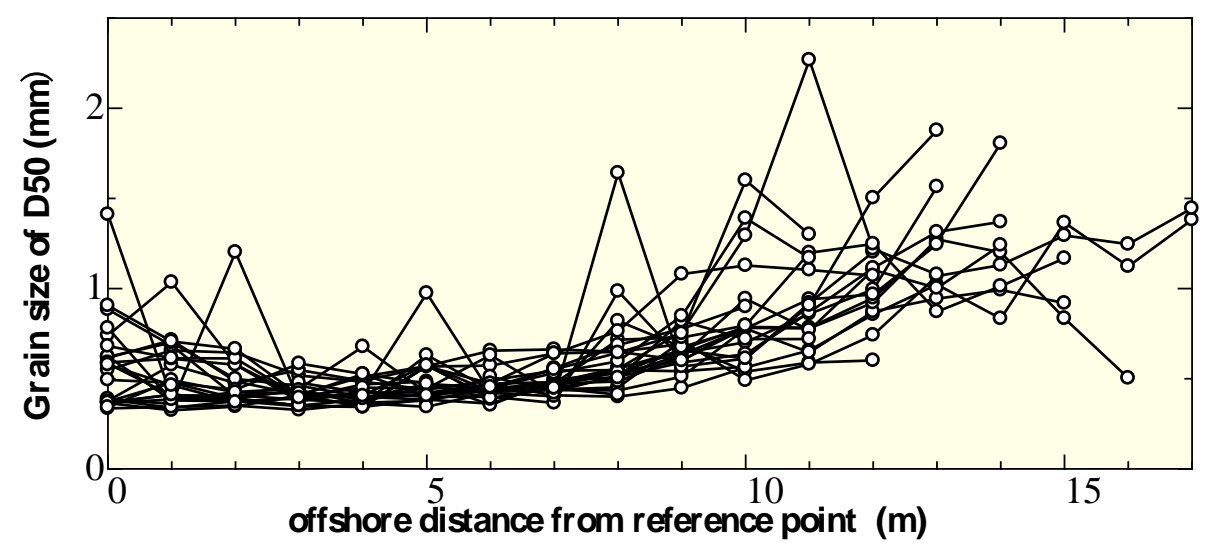

Fig. 3 Cross-shore grain size distributions

\section{Analysis method}

(1) Quadratic profile approach

To evaluate temporal changes of cross-shore profiles at Okoshiki Beach, Yamada et al. (2004) used a quadratic polynomial equation fitted to measured beach profiles, proposing three parameters. Here, derivation of the parameters is described briefly. The quadratic equation fitted to the measured profiles is expressed as follows.

$$
z(t, x)=-a(t) x^{2}-b(t) x+c(t),
$$

where $\mathrm{z}$ is measured bed level (defined as positive upward), $\mathrm{t}$ is month, and $\mathrm{x}$ is offshore distance. Parameter $a$ is convexity that has a positive upward profile, $b$ is local slope at $\mathrm{x}=0$ that is positive downward, and $c$ is bed elevation at $x=0$. These parameters are assumed to dependent on $t$ alone. In addition, $\mathrm{z}$ is median grain size when applying Eq. (1) to the grain size distributions.

To evaluate agreement quantitatively, we use the average deviation $\varepsilon$ between measured and fitted profiles, defined as 


$$
\varepsilon=\frac{1}{N} \sum_{n=1}^{N}\left|z_{n}-\left(-a x_{n}{ }^{2}-b x_{n}+c\right)\right|,
$$

where $\mathrm{N}$ is the total number of data points along survey lines in the region $\mathrm{x}=0-18 \mathrm{~m}$, for each measured profile. The fitted profile agrees with measured profiles within an error of $3 \mathrm{~cm}$ for bed level profile, and $0.1 \mathrm{~mm}$ for sand grain size.

Quadratic convexity $Q$, mean slope $S$ and vertical displacement $D$ are introduced here to interpret temporal parameters $a, b$ and $c$, because $b$ and $c$ characterize the quadratic profile at $\mathrm{x}=0$ as previously mentioned. These parameters are given by

$$
\begin{aligned}
& Q=a\left(\frac{x_{2}-x_{1}}{2}\right)^{2} \\
& S=a\left(x_{1}+x_{2}\right)+b \\
& D=-\frac{\left(a-a_{m}\right)}{3}\left(x_{1}^{2}+x_{2}^{2}+x_{1} x_{2}\right)-\frac{\left(b-b_{m}\right)}{2}\left(x_{1}+x_{2}\right)+\left(c-c_{m}\right)
\end{aligned}
$$

where $x_{1}$ and $x_{2}$ are landward and seaward ends of the survey line, respectively, $a_{m}, b_{m}$ and $c_{m}$ are coefficients for time-averaged profiles. Subscript "d" is appended for sand grain size. Concavity of grain size distribution $Q d$, sorting $S d$ and displacement from mean grain size $D d$ are now introduced. Table 1 shows the range of values for $Q, S, D, Q d, S d$ and $D d$.

\begin{tabular}{|c|c|c|c|c|c|c|}
\hline \multicolumn{6}{|c|}{ Table-1 Six parameters for beach profile and grain size } \\
\hline & \multicolumn{3}{|c|}{ Beach profile } & \multicolumn{3}{|c|}{ Grain size distribution } \\
\cline { 2 - 7 } & $\mathrm{Q}(\mathrm{cm})$ & $\mathrm{S} * 10^{-1}$ & $\mathrm{D}(\mathrm{c} \mathrm{m})$ & $\mathrm{Qd} * 10^{-2}(\mathrm{~mm})$ & $\mathrm{Sd} * 10^{-1}$ & $\mathrm{Dd} * 10^{-1}(\mathrm{~mm})$ \\
\hline Maximum & 35.9 & 1.65 & 23.4 & -89.3 & 0.55 & 2.44 \\
\hline Minimum & -9.94 & 1.15 & -29.0 & 8.5 & -9.33 & -1.79 \\
Mean & 10.7 & 1.37 & 0 & -36.1 & -4.55 & 0.13 \\
\hline
\end{tabular}

(2) Principal component analysis and variation characteristics of beach profiles and sand particle size distributions

As explained previously, there are three parameters for beach profiles and grain size distributions. Principal component analysis (PCA) was applied to the six parameters, to examine relationships between their fluctuations and discover new variables that effectively express overall fluctuation of the parameters. There are a variety of external forces that influence the beach profiles and sand particle size distributions, so relationships between their fluctuation and these external forces were examined by multiple regression analysis. The six parameters have their own units, and we used PCA with a standardized covariance matrix.

Results show that the first and second axes (PC1 and PC2) extracted by PCA together accounted for

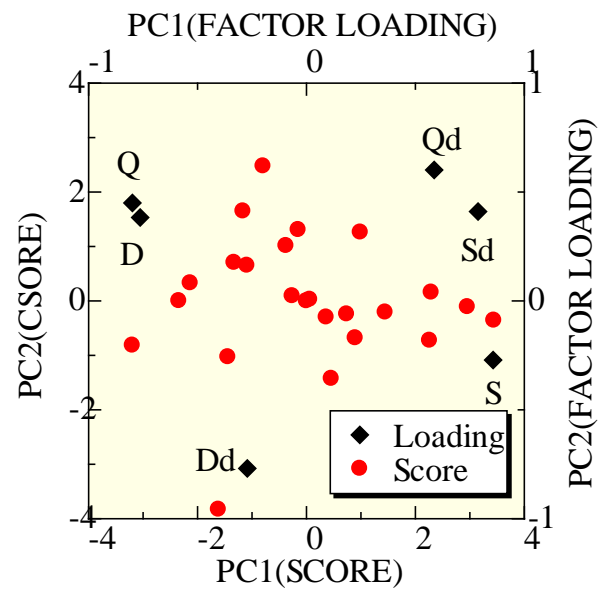

Fig. 4 Factor loadings of six parameters and first two principal component cores 
$76 \%$ of total data variance. PC1 (eigenvalue=2.99) accounted for 50\% of the variance and PC2 (eigenvalue $=1.54$ ) $25.7 \%$, respectively. Therefore, the first two components are used hereafter to analyze measured data. Figure 4 shows factor loadings of the six parameters and scores of the 23 observations, plotted along the first two principal component axes. Variables $S, Q d$, and $S d$ had significantly positive loadings and $Q, D$ and $D d$ negative ones on the PC1 axis. Therefore, an increase of PC1 score indicates an increase of beach profile slope $S$ and decrease of average level $D$ and curvature of profile $Q$. Values of $Q d$ and $S d$ have positive loadings and $D d$ negative ones for PC1 axis. The value of $S d$ was less than zero for all 23 observations and consequently grain size continuously increases in the cross-shore direction. Figure 5 shows relationships among beach profiles, grain size distributions and PC score.

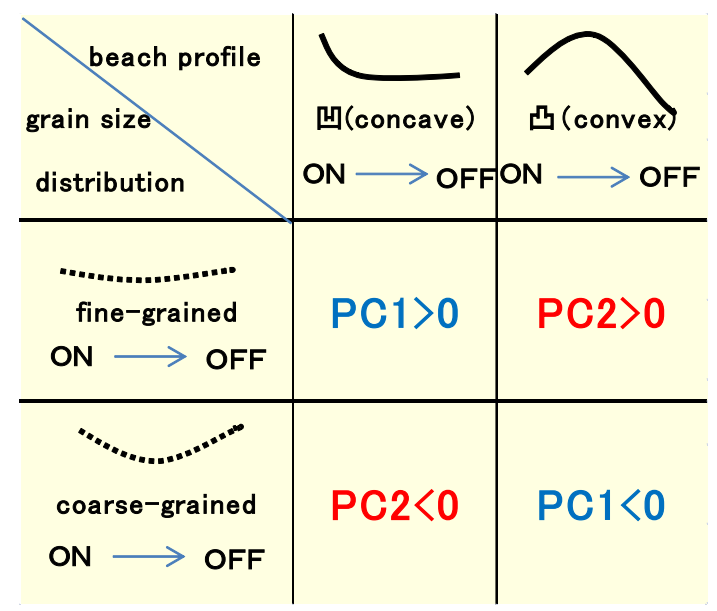

Fig. 5 Classification of beach profiles and grain size distributions

\section{Results and discussion}

(1) Determining factors and prediction method of overall fluctuation patterns

Both tidal level and wave height are expected to influence changes of beach profiles and grain size distributions. Therefore, multiple regression analyses were done to examine relationships between overall fluctuation patterns (variations of principal component scores) as dependent variables, and statistical, independent variables. These variables include average and maximum tidal levels and wave heights between consecutive observations, averages over 3 or 7 days before each observation, and others.

The multiple regression analysis was executed on data from all 23 observations to find significant independent variables (Run 1), and on data from the first half of these observations to get regression coefficients for the prediction calculation (Run 2). This calculation for the latter (period of observations 12 through 23) was done using regression coefficients of significant independent variables obtained from Runs 1 and 2 .

Table 2 shows results of the regression analyses. Run 1 indicates that average tidal level over 7 days prior to each observation th7, and average of daily maximum significant wave height between two consecutive observations H1/3 $3_{\max }$ are the most significant factors for scores PC1 and PC2, respectively. Run 2 reveals that although there is significant correlation between PC2 scores and H1/3 $3_{\max }$, all regression coefficients of the run resemble those of Run 1 . Therefore, coefficients of Run 2 were used for the prediction calculation. 


\begin{tabular}{|c|c|c|c|c|c|c|c|}
\hline Run & $\begin{array}{l}\text { PC } \\
\text { No. }\end{array}$ & $\begin{array}{c}\text { Analized } \\
\text { Obs. }\end{array}$ & Variables & Coefficeints & T-values & $\mathrm{P}$-values & Significance \\
\hline \multirow{4}{*}{1} & \multirow{2}{*}{1} & \multirow{2}{*}{$1-23$} & th7 & -9.6 & -3.3 & 0.003 & $* \quad *$ \\
\hline & & & constant. & 15.2 & 3.3 & 0.003 & $* \quad *$ \\
\hline & \multirow{2}{*}{2} & \multirow{2}{*}{$1-23$} & $\mathrm{H} 1 / 3 \max$ & 7.2 & 2.8 & 0.011 & * \\
\hline & & & constant. & -1.9 & -2.7 & 0.015 & $*$ \\
\hline \multirow{5}{*}{2} & \multirow{2}{*}{1} & \multirow{2}{*}{$1-12$} & th7 & -12.6 & -3.4 & 0.007 & $* \quad *$ \\
\hline & & & constant. & 20 & 3.4 & 0.007 & $* *$ \\
\hline & \multirow{2}{*}{2} & \multirow{2}{*}{$1-12$} & $\mathrm{H} 1 / 3 \max$ & 6.8 & 1.2 & 0.26 & \\
\hline & & & constant. & -1.8 & -0.9 & 0.383 & \\
\hline & & & & gnifican & el $1 \% *$ & nificant & el $5 \%$ \\
\hline
\end{tabular}

Results of the prediction calculation for the latter half of observations are shown in Fig. 6. Horizontal and vertical axes are observation numbers for the two principal component scores. Predicted scores of these components reproduce the time series variation on a scale about one year of the observed scores, but not those with time scales less than one year.
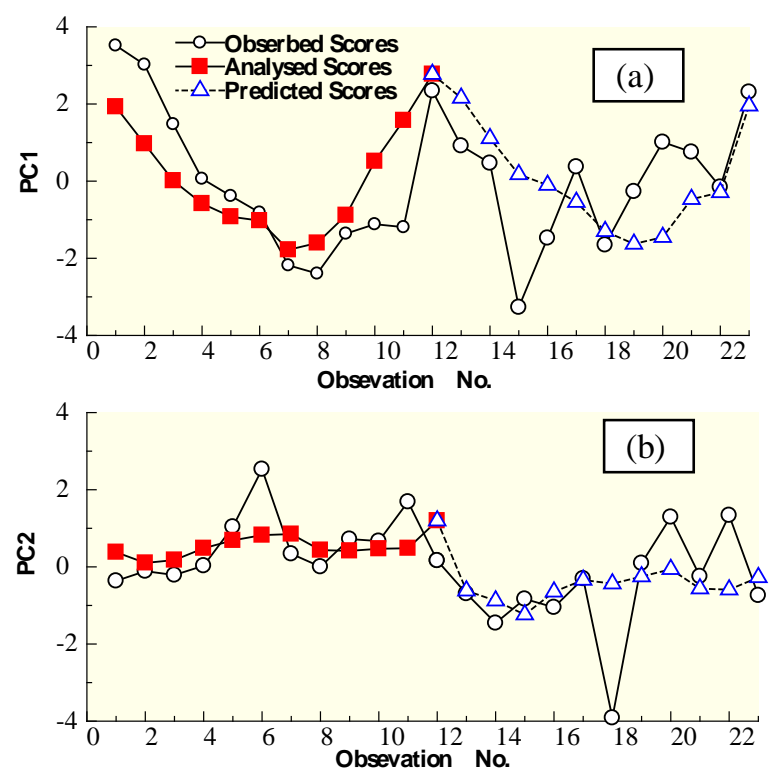

Fig. 6 Time series of observed, analyzed and predicted scores

\section{(2) Long-term prediction method}

To obtain a long-term prediction method, the first two PC scores expressing overall fluctuation patterns were calculated for every month of the ten years from 2011 - 2020. The th7 was predicted using tidal harmonic constants at the port of Akashi, which is about $4 \mathrm{~km}$ west of the field site. The H1 $/ 3_{\max }$ was predicted by a Monte Carlo simulation, in which wind direction and speed were randomly generated. Both annual and seasonal frequency distributions of wind direction and speed were estimated using the last two years of wind observations at Akashi.

Figure 7(a) and (b) shows annual frequency distributions of wind direction and speed as an example. The horizontal axis in Fig. 7(a) includes the 16 cardinal directions, in which number 1 represents a NNE direction, and direction increases clockwise by 22.5 degrees per unit. The number 0 means calm conditions or a wind speed below measuring capabilities. Frequency distributions of wind speed are often represented by the Weibull distributions: 


$$
f(x)=\frac{\alpha}{\beta^{\alpha}} x^{\alpha-1} e^{-(x / \beta)^{\alpha}},
$$

where $X$ is wind speed and $\alpha$ and $\beta$ are parameters. The parameters are estimated using wind data from Eigashima port. Figure 7(b) demonstrates that the Weibull distribution ( $\alpha=1.3, \quad \beta=3.8$ ) agrees well with the observed annual frequency distribution.

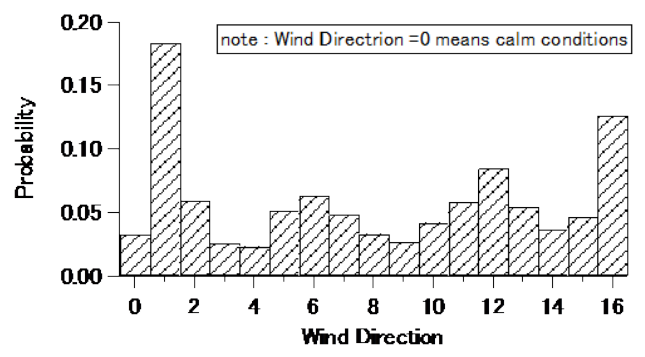

(a)Wind directions

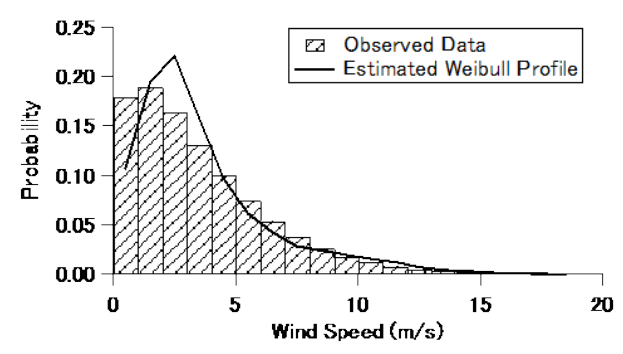

(b)Wind speed and Weibull distribution

Fig. 7 Annual frequency distributions of wind

A uniformly distributed random number between 0 and 1 is generated and substituted into the inverse function of the Weibull distribution. This obtains time series of wind speed of whose probability distribution agrees with the Weibull distribution of Eq. (5) for long-term prediction calculations. To attain random wind direction sequences that agree with observed frequency distributions, a uniformly distributed random number between 0 and 1 was also generated, and wind direction determined by the following condition:

$$
\text { Wind Direction }=\left\{\begin{array}{lr}
0 & \text { when } r \leq p_{0} \\
n & \text { when } \sum_{i=1}^{n} p_{i-1} \leq r<\sum_{i=1}^{n+1} p_{i-1}
\end{array},\right.
$$

where $\mathrm{p}_{\mathrm{i}}$ : relative frequency of the $\mathrm{i}^{\text {th }}$ wind direction and $\mathrm{r}$ is a random number.

Fetches of the 16 wind directions must be determined to calculate significant wave height H1/3. Figure 8(a) compares these fetches measured by a topographic map (Fm) with fetches of 7 directions (out of the 16) calculated by observed wave height at Eigashima port, $F w$. Since values of $F m$ and $F w$ generally agree, $\mathrm{Fm}$ is hereafter used as the maximum fetch for each direction. Assuming a wind duration of $1 \mathrm{~h}$, fetches are also calculated by multiplying the randomly generated wind speed and assumed wind duration. These fetches are called Fs. After comparing Fw and Fs, the shorter of the two was selected to calculate H1/3 in the long-term prediction.

Figure 8(b) compares the calculated H1/3 using a simple prediction method proposed by Goda (2002) with observed data at Eigashima port. The line in Fig.8 (b) shows a regression line with gradient 0.6 and determination coefficient 0.54 . The calculated significant wave height $\mathrm{H} 1 / 3$ tends to be underestimated by $40 \%$.

Figure 9 (a) shows time series of predicted PC1 scores for the ten years. They fluctuate between -2 to 2, with maxima in Decembers and minima in Augusts. They seem to reproduce characteristics of fluctuation in component PC1, with time scale about 1 year (Fig.6). This means that the beach profiles changed from an erosive to depositional type. In addition, the sediment prosperity sediment changed 

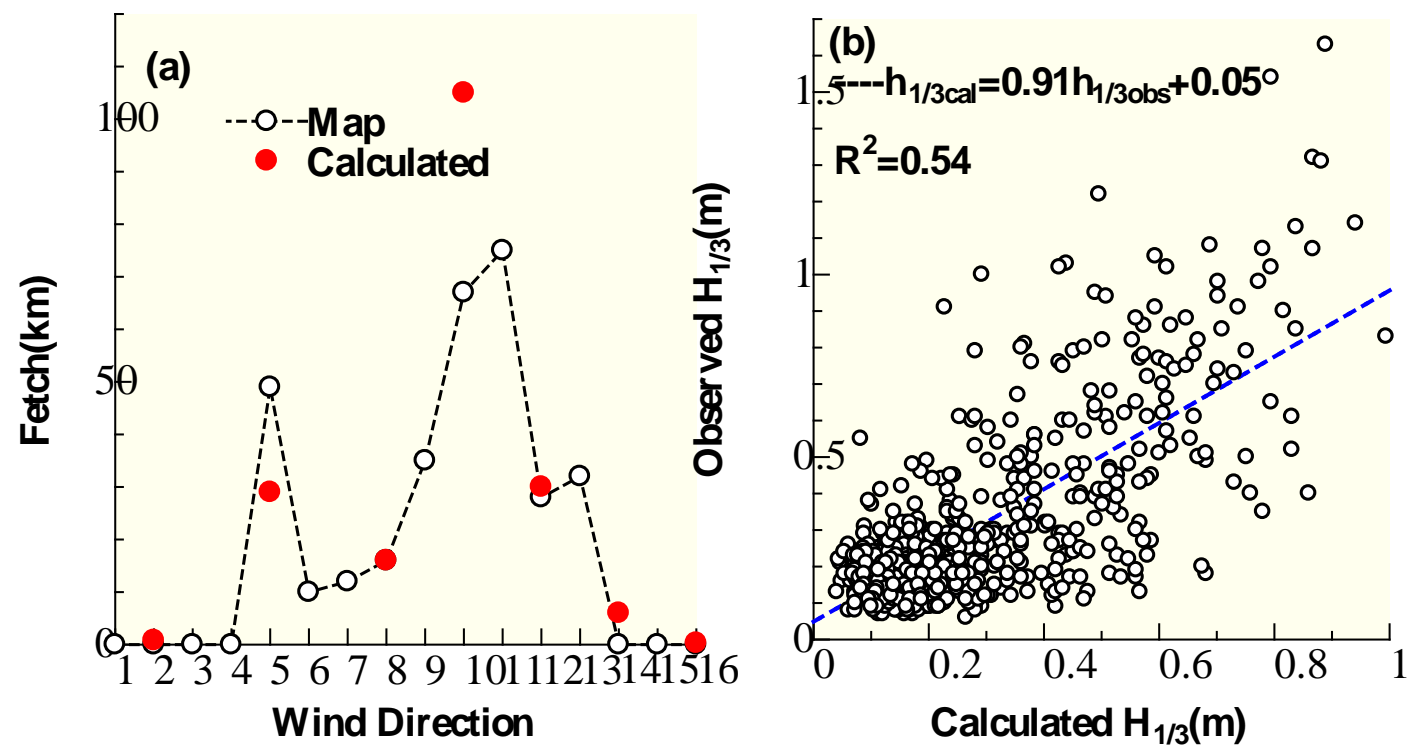

Fig. 8 Fetches of 16 cardinal directions and comparisons between calculated and observed $\mathrm{H}_{1 / 3}$. .

from fine-grain to coarse sand between winter and summer each year, because of variations in high tide level.

First, predicted PC2 scores were calculated with annual frequency distributions of wind direction and speed and regression coefficients of Run 2(listed in Table 2). This is termed Case1. Time series of PC2 scores for this case are shown in Fig. 9(b). The range of score variation is 0.5 to 1.4, inconsistent with the observed one shown in Fig. 6(b); this is because the calculated wave height for this case is much smaller than observed. Second, seasonal frequency distributions of wind direction and speed were constructed for calculations of significant wave height. This is termed Case 2. The predicted PC 2 scores for this case (Fig. 9(b)) fluctuate with amplitude about 1.5, which is approximately equal to that of the observed PC 2 scores in Fig. 6(b). Consequently, we infer that score fluctuation is chiefly caused by seasonal variations of wind direction and speed. The observed PC2 scores suddenly dropped, and the erosion process evolved in winter as displayed in Fig. 6(b). The sudden drop was caused by low significant wave height. However, the predicted scores for Case 2 gradually decrease from late winter to autumn, and suddenly increased in winter. This tendency is inconsistent with the observed one in Fig. 6 (b). Northwest winds are frequent and strong in winter, but $F m$ for this direction is very short (Fig. 8). Therefore, a small predicted small H1/3 $3_{\max }$ increased PC scores in Case2. We are

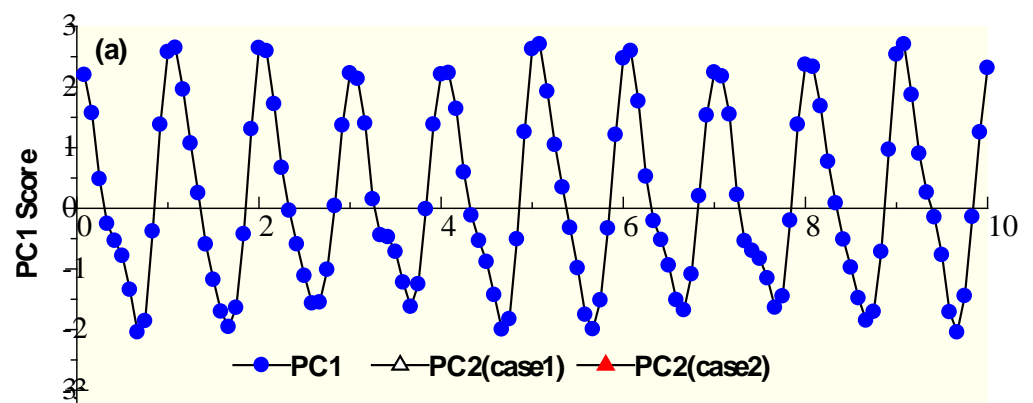

(b)

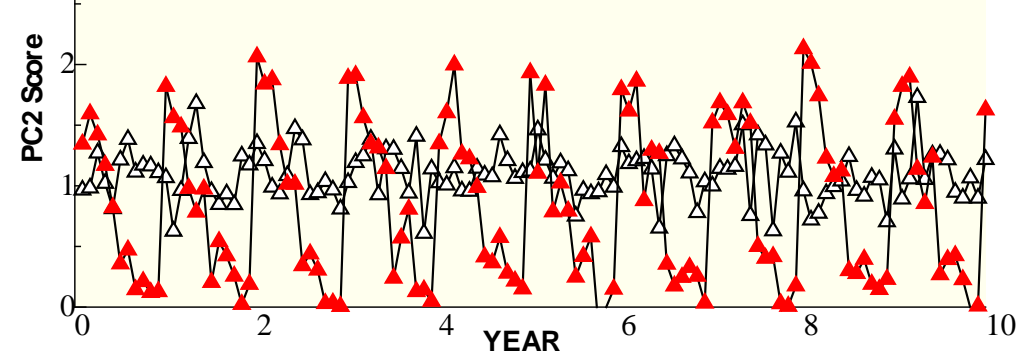

Fig. 9 Time series of long term prediction for PC1 and PC2 scores 
conducting further observations and analysis to examine the inconsistency in fluctuation phase between the observed scores and long-term prediction.

\section{CONCLUSIONS}

Focusing on seasonal variations of beach characteristics at Azure Maiko Beach, the static approach was applied to cross-shore beach profiles and sand grain size distributions. The following conclusions were obtained.

(1) Three new parameters for sand grain size distributions were proposed, and variation patterns of beach profiles and sand grain distributions were clarified using PCA.

(2) The first two components of PCA were able to express characteristics of Azure Maiko Beach. PC1 is related to an average tidal level over the 7 days prior to each observation. PC2 is associated with an average daily maximum significant wave height between consecutive observations.

(3) Change of sediment properties is strongly subject to the seasonal wave variation rather than tide level.

(4) Long- term prediction of PC1 scores was be simulated well. However, improvement of wave forecasting and hind-casting is necessary for long- term prediction of PC2 scores.

\section{ACKNOWLEDGMENTS}

Observed wave data were obtained from the Ministry of Land, Infrastructure, Transport and Tourism. A part of this study was supported by the Grant in Aid for Science Research Ministry of Education, Culture, Sports and Technology.

\section{REFERENCES}

Goda, Y. 2002. A simple wind wave estimation based on Wilson's prediction formulas, ECOH/YG Technical Report No.1.

Jackson, N. L., K. L. Nordstrom, I. Eliot and G. Masseilink 2002. Low-energy sandy beaches in marine and estuarine environments; a review, Geomorphology, Vol.47, pp.147-162

Tsujimoto Gozo, F. Yamada, D. Sakai, H. Kaida and T. Kakinoki 2010. Seasonal variation of crossshore beach profile with a filter layer, Annual Journal of Civil Engineering in the Ocean, JSCE, Vol.26,pp.1209-1214 (in Japanese).

Yamada Fumihiko and N. Kobayashi 2004: Annual variations of tide level and mudflat profile, Journal of Waterway, Port, Costal and Ocean Eng., ASCE,Vol.130,No.3,pp.119-126

Rubin D. M. 2004. A simple autocorrelation algorithm for determining grain size from digital image of sediment, Journal of Sedimentary Research, Vol.74, No.1,pp.160-165 